\title{
Parametric Adaptive Spectrum Sensing Framework for Dynamic Spectrum Access Networks
}

\author{
Dinesh Datla \\ Rakesh Rajbanshi \\ Alexander M. Wyglinski \\ Gary J. Minden \\ Information and Telecommunication Technology Center \\ The University of Kansas, Lawrence, KS 66045 \\ Email: \{dineshd, rajbansh, alexw, gminden\}@ittc.ku.edu
}

\begin{abstract}
The opportunistic usage of the spectrum must be done without causing any interference to the licensed spectrum users. Conventional non-adaptive wideband spectrum sensing approaches could potentially be inefficient since they generally employ the same scanning resolution, even though the spectrum might contain different types of signals, individually requiring scans with different resolutions. In this paper, we present a novel spectrum sensing framework that adapts its parameters across the spectrum of interest according to the characteristics of its occupancy. We also propose a dynamic scheduling algorithm for spectrum sensing which allocates different time resolutions to different portions of the spectrum. We demonstrate that the proposed algorithm improves the efficiency of spectrum sensing over a non-adaptive approach.
\end{abstract}

\section{Introduction}

Dynamic spectrum access (DSA) networks promise to solve the spectrum scarcity problem by improving the spectrum utilization. However, implementation of the DSA approach over a wide bandwidth is not trivial. The agile radio may potentially encounter primary users that possess different transmission characteristics that vary as a function of frequency, time, and space [1]. In order to avoid interference to the primary users, the radio should be capable of adapting to these changes as well as dynamically sense the spectrum over a wide bandwidth and detect the presence of signals with a low probability of interception [2].

Conventional approaches to wideband spectrum sensing possess several limitations. First, sensing the entire target spectrum continuously may be inefficient. Frequency channels with a high occupancy rate need not be sensed frequently, while channels with a high channel availability need to be monitored more frequently. Second, the channel occupancy state can change rapidly such that the sensing mechanism may fail to keep track of the instantaneous states due to limitations on the sampling time resolution. Such a situation can result in an inaccurate characterization of the spectrum occupancy.

In this paper, we address these issues with the proposed Parametric Adaptive Spectrum Sensing (PASS) architecture for dynamic spectrum sensing. In our approach, the radio learns the statistics of the channel availability by building an updated model of the channel occupancy, while the spectrum sensing mechanism adapts its parameters

This work was supported by NSF grants ANI-0230786 and ANI0335272 . based on this model. Furthermore, we propose an algorithm for efficient scheduling of the sensing mechanism's time-frequency assignments. While there has been some preliminary work done on channel-aware spectrum sensing techniques [3], the sensing in [3] is performed at fixed intervals, while our algorithm varies the sensing schedule dynamically according to the channel occupancy statistics.

The rest of the paper is organized as follows: Section 2 presents the proposed spectrum sensing architecture as well as an introduction to the parameters of the sensing mechanism. The proposed scheduling algorithm is presented in Section 3 followed by simulation results in Section 4 . The concluding remarks are presented in Section 5 .

\section{Proposed Parametric Adaptive Spectrum Sensing Architecture}

The proposed PASS architecture enables the radio to adapt its spectrum sensing mechanism to variations in the spectrum occupancy. The block diagram for the proposed PASS architecture is shown in Fig. 1.

During the sensing operation, the $R F$ front end functions as a passive receiver that captures the spectrum measurements, with the signal presence detected using energy detection techniques[4]. The spectrum sensing control mechanism controls the parameters of the sensing operation. It translates the requirements of the sensing mechanism into the parameters of the RF components, such as the local oscillator's center frequency. The $R F$ front end and the spectrum sensing control block constitute the cognitive radio's spectrum sensing mechanism. The data management and processing (DMP) block maintains a database of the spectrum measurements that are either collected by the radio itself or obtained from other radios in a collaborative spectrum sensing setup [5]. The data from the database is retrieved for processing to detect the presence of signals.

The characterization of the channel occupancy is important to identify potential opportunities for secondary access. The characterization and learning block performs the analysis on the processed measurement data and characterizes the radio's operating spectrum. It can obtain the processed data from the DMP block and use it to build a spectrum occupancy model, as well as update the parameters of the model. In this paper, we assume a Markov model with states 'channel occupied' and 'channel available' represented by ' 0 ' and ' 1 '. The radio's learning process occurs in two stages: initial learning and simultaneous learning. Initial learning refers to the stage of learning 


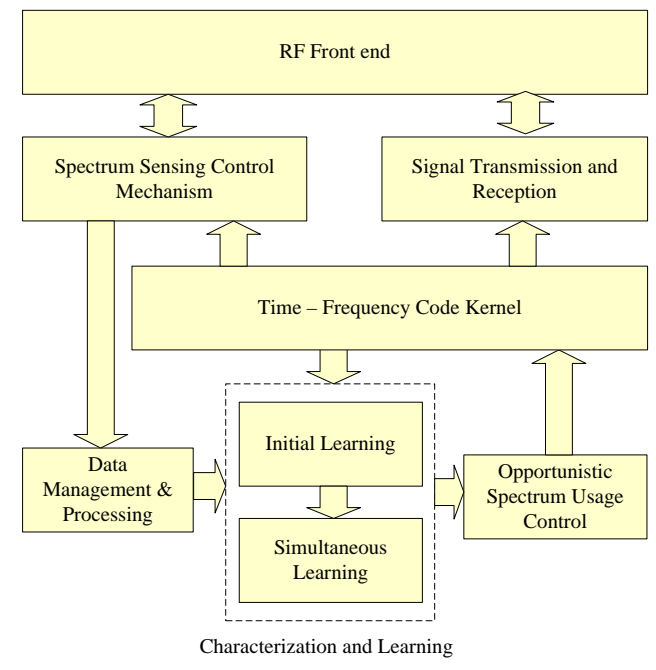

Fig. 1 Block Diagram of the proposed Parametric Adaptive Spectrum Sensing architecture.

when the radio is deployed and it learns its wireless environment before becoming fully-functional. In this stage, the model parameters are estimated from the measurement data. When the estimates of the parameters reach a certain confidence level, initial learning stops and the radio becomes fully-functional. At this point, the stage of simultaneous learning begins. In this stage, the $R F$ front end is shared by the sensing mechanism and the radio communication subsystem, and the learning process occurs simultaneously along with the primary operations of the radio ${ }^{1}$. As more data is collected and analyzed, the parameters of the model are updated. The statistics on channel availability are made available to the opportunistic spectrum usage (OSU) control block.

The OSU block may be implemented in the radio resource management layer of a DSA network. The $O S U$ control block chooses a set of frequency channels among a pool of unoccupied channels based on the wireless channel characteristics, QoS requirements, and regulations for spectrum access. Accordingly, it decides on which channels to sense and at what time intervals to sense them. Thus, the OSU block decides the time-frequency assignments for the sensing operations, and radio communication operations. The time-frequency code kernel block stores these assignments.

The spectrum sensing mechanism adapts its sweep parameters and scanning assignments according to the estimated model of the spectrum occupancy, hence the name parametric adaptive spectrum sensing.

We consider the use of a superheterodyne receiver-based spectrum sensing mechanism to measure the power distribution in a certain frequency band. During a measurement test, the measuring instrument scans across the sweep bandwidth $B_{s}$ in steps of $B_{r}$, where $B_{r}$ is the bandwidth resolution. Sweep time $T_{s}$ is the total time taken to complete a single sweep over the bandwidth $B_{s}$. The mea-

\footnotetext{
${ }^{1}$ Sensing operations involve scanning for spectral white spaces and collecting data for characterizing spectrum occupancy, and radio communication operations include transmission and reception of information-bearing signals.
}

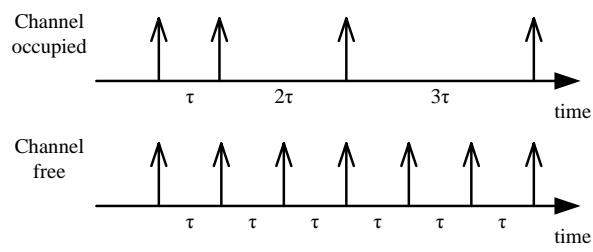

Fig. 2 Backoff in sweep time resolution.

surement test is conducted over a certain period of time as specified by the measurement test duration $T$. The instantaneous power residing in a certain frequency channel is measured at specific time intervals as specified by the sweep time resolution $T_{r}$. Thus, the sweep time resolution specifies the time elapsed between two consecutive power measurements of a certain frequency channel, which implies that the sweep time resolution is directly proportional to the sweep time itself.

\section{Proposed Scheduling of Spectrum Sensing Assignments}

An efficient scheduling of the time-frequency assignments of the spectrum sensing mechanism would improve the time resolution of the sweeps besides increasing the efficiency of the spectrum sensing. We present a novel algorithm that schedules the sweeps both along time and frequency, based on the channel occupancy statistics. The underlying principle is that the spectrum sensing mechanism invests more time with frequency channels that have a high probability of channel availability, thereby making the algorithm adaptive.

The algorithm performs a backoff in the time resolution every time the channel is detected to be occupied. The backoff can be linear, or exponential. Fig. 2 illustrates the linear backoff in time resolution for the case when the channel is always occupied and for the case when it is being unutilized throughout the observation time.

The underlying idea is to minimize the number of scanning assignments and avoid wasteful sensing over presumably occupied segments of the spectrum. Minimizing the scanning assignments can reduce the sweep time. In this way, by reducing the sweep time, better time resolution can be provided to channels that are available with high probability. The details of the proposed algorithm are presented next.

Let $N_{c}$ be the number of channels required by the radio for transmission. Depending on the time-bandwidth requirements of the radio and the availability of the same in the spectrum, $N_{c}$ can be varied over time. For our discussion, we assume $N_{c}$ is fixed, with no specific requirement on the time duration of the channel availability. $N_{c}$ number of channels with the highest probability of availability $P_{0}^{i}$ are selected and we can represent them as a set of channels $\left\{f_{i}\right\}, i=1,2 \ldots N_{c}$. These channels may not be contiguous in frequency. For the $i^{t h}$ channel, the sweep time resolution is expressed as $n_{i} \tau$ which is a multiple of the fundamental sample time $\tau$ and $n_{i}$ is a variable scaling factor. The time-frequency assignments for the spectrum sensing mechanism are stored in matrix $\mathbf{A}=\left[A\left(f_{i}, t_{j}\right)\right]$ where $A\left(f_{i}, t_{j}\right)$ specifies if the radio has to scan channel $f_{i}$ at time instance $t_{j}$. 


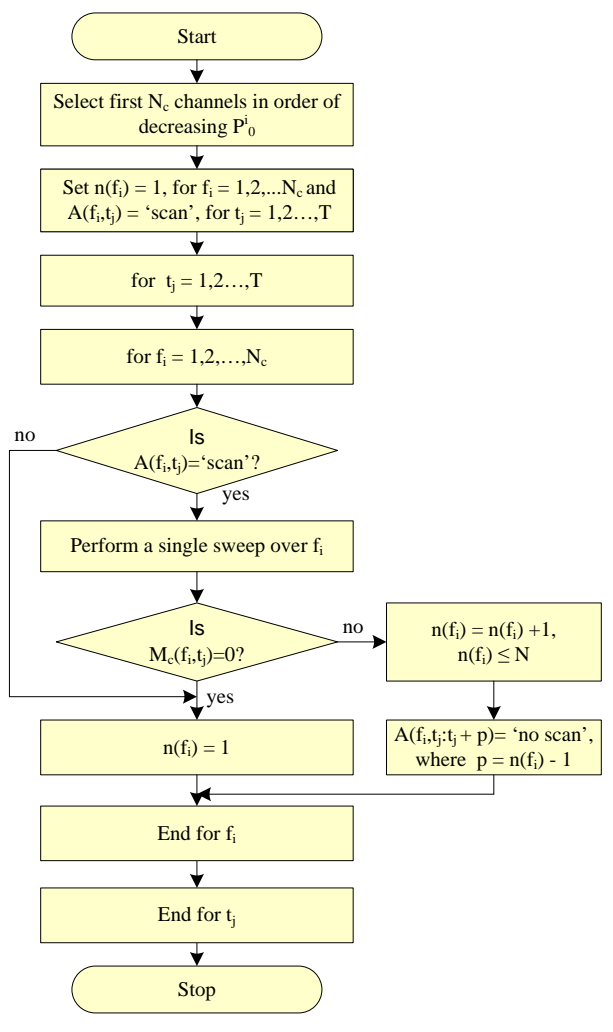

Fig. 3 Flowchart of proposed scheduling algorithm.

Fig. 3 shows the flowchart for the proposed algorithm for a linear backoff case. We assume a total time of $T$ for our simulation. Initially, the sensing mechanism is assigned to scan all the frequency channels $\left\{f_{i}\right\}$ at all time instants $t_{j}=1,2, \ldots, T$ and all the values in $\mathbf{A}$ are set to 'scan'. During each time instance $t_{j}$, before scanning the frequency channels, the sensing mechanism first reads from the corresponding row in $\mathbf{A}$ and scans only those channels that have been scheduled for sensing. After sensing, based on the perceived channel occupancy state $M_{c}\left(f_{i}, t_{j}\right), n_{i}$ is either incremented or set to the default value of 1 . Accordingly the sensing schedule for the frequency channels at future time instances is updated in $\mathbf{A}$. The maximum backoff $N$ can be specified. Incrementing $n_{i}$ results in backoff in the time resolution for sensing channel $f_{i}$.

\section{Simulation}

\subsection{Simulation Setup}

In our computer simulation, we randomly generated the spectrum occupancy in frequency channels considering an ideal TV band spectrum with a bandwidth of $33 \mathrm{MHz}$ and a $10 \mathrm{kHz}$ spacing between adjacent spectral samples. In the simulated TV spectrum, all the TV channels are assumed licensed and the stations stay 'on' throughout the observation time. Each TV station occupies $6 \mathrm{MHz}$ of the spectrum which also includes the guard bands. The guard bands between adjacent TV stations are occupied by secondary users that transmit in bursts. The bursty signals have exponentially distributed 'on' times, where the mean 'on' time is randomly selected from a uniform

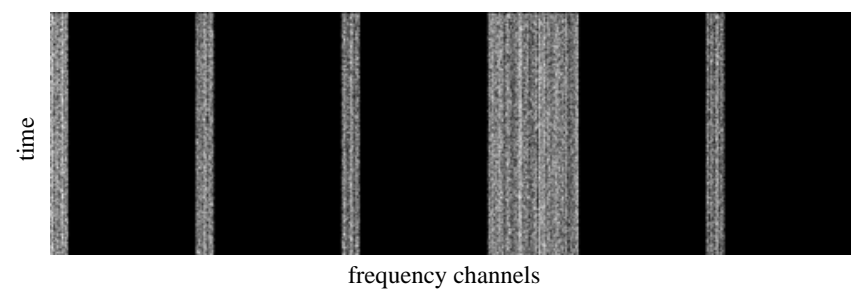

Fig. 4 Intensity plot of simulated occupancy values for ideal TV band. The darker areas represent the occupied spectrum.

distribution on the interval $(0.0,1.0)$. We generated 1000 instances of the TV spectrum occupancy. Each channel has different mean 'on' times and different channel occupancy statistics and this allows us to exploit the adaptive nature of the proposed algorithm. It is further assumed that the channel occupancy state does not vary over the sampling intervals. Fig. 4 shows the intensity plot for the simulated spectrum occupancy. In the figure, the darker areas represent the occupied spectrum while the unoccupied spectrum is represented by the bright regions. The proposed scheduling algorithm using a linear backoff was run over this randomly generated TV spectrum. To test the algorithm in the worst case scenario, it was assumed that $N_{c}=33 \mathrm{MHz}$, which means that all the channels are considered for scanning initially including the TV station channels with $100 \%$ occupancy.

\subsection{Simulation Results}

Fig. 5(a) shows the proportion of the total number of channels that were sensed during each time instance and Fig. 5(b) shows the proportion of the total time for which each channel was sensed. If the conventional fixed scheduling approach was followed, ideally all the channels should have been sensed $100 \%$ of the time. However, by applying the proposed algorithm, it is seen from the results that there is a drastic decrease in the sweep time at each time instance (Fig. 5(a)) and over the occupied bands the sensing mechanism scans for only a very small percentage of the total observation time Fig. 5(b). The portions of the spectrum occupied by the TV signals are scanned with a low time resolution with large spacing between consecutive time samples. The sweep time resolution is high for the frequency channels that are sparsely occupied and the resolution varies according to the spectrum occupancy in each of those channels. The simulation was repeated 100 times and it was determined that, on an average, there is a $84.23 \%$ improvement in the efficiency over the conventional approach. However, on an average, $1.84 \%$ of the potential channel vacancies were not scanned due to the backoff, which is insignificant when compared to the drastic improvement in the sensing efficiency. This improvement in efficiency reflects as savings in time and power consumption, assuming that the sensing mechanism is switched off when it is not assigned to scan.

\section{Conclusions}

This paper introduced the PASS framework for efficient and fast opportunistic spectrum access by spectrum sensing, channel occupancy modeling and adapting the spec- 


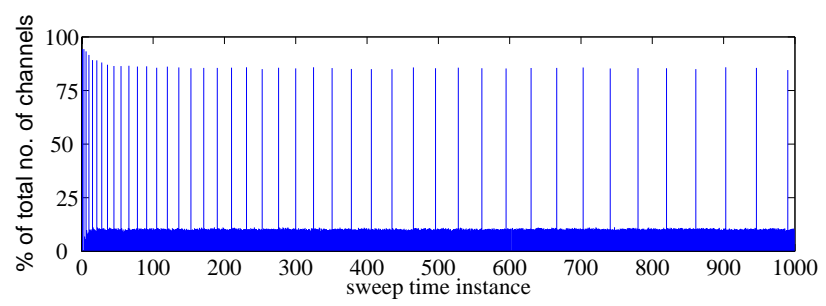

(a) Number of channels sensed at each time instance.

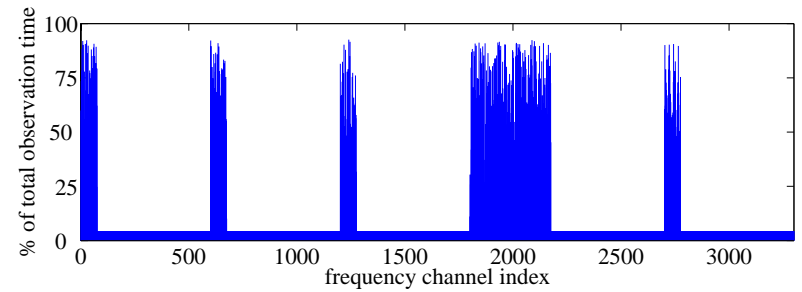

(b) Total sensing time for each channel.

Fig. 5 Simulation results for linear backoff when applied to ideal TV spectrum shared with secondary signals.

trum sensing parameters based on the estimated channel occupancy model. By following a channel-aware adaptive approach, the PASS framework can overcome the drawbacks of the conventional non-adaptive sensing mechanisms such as limited time resolution.

We also presented a novel algorithm for scheduling the assignments for spectrum sensing both over frequency and time. The simulation results showed a significant improvement in the sensing efficiency as compared to a conventional non-adaptive approach.

\section{References}

[1] A. J. Petrin, Maximizing the Utility of Radio Spectrum: Broadband Spectrum Measurements and Occupancy Model for Use by Cognitive Radio. PhD thesis, School of Electrical and Computer Engineering, Georgia Institute of Technology, August 2005.

[2] D. Cabric, S. M. Mishra, and R. W. Brodersen, "Implementation issues in spectrum sensing for cognitive radios," in Conference Record of the Thirty-Eighth Asilomar Conference on Signals, Systems and Computers, vol. 1, (Pacific Grove, California, USA), pp. 772- 776, Nov. 2004.

[3] S. Jones, N. Merheb, and I.-J. Wang, "An experiment for sensingbased opportunistic spectrum access in CSMA/CA networks," in IEEE International Symposium on New Frontiers in Dynamic Spectrum Access Networks, (Baltimore, MD, USA), pp. 593- 596, November 2005.

[4] F. Weidling, D. Datla, V. Petty, P. Krishnan, and G. J. Minden, "A Framework for RF Spectrum Measurements and Analysis," in IEEE International Symposium on New Frontiers in Dynamic Spectrum Access Networks, (Baltimore, MD, USA), pp. 573-576, November 2005.

[5] Amir Ghasemi and Elvino Sousa, "Collaborative Spectrum Sensing for Opportunistic Access in Fading Envionments," in IEEE International Symposium on New Frontiers in Dynamic Spectrum Access Networks, (Baltimore, MD, USA), pp. 131-136, November 2005. 